Marta Corrêa Dalbem

\title{
Análise de Investimentos em Energia Eólica no Brasil
}

\section{Tese de Doutorado}

Tese apresentada ao Programa de Pós-Graduação em Administração de Empresas da PUC-Rio como requisito parcial para obtenção do título de Doutor em Administração de Empresas.

Orientador: Prof. Leonardo Lima Gomes

Co-Orientador: Pedro Americo Moretz-Shon David

Rio de Janeiro

Setembro de 2010 
Marta Corrêa Dalbem

\title{
Análise de Investimentos em Energia Eólica no Brasil
}

Tese apresentada ao Programa de Pós-Graduação em Administração de Empresas da PUC-Rio como requisito parcial para obtenção do título de Doutor em Administração de Empresas da PUC-Rio. Aprovada pela Comissão Examinadora abaixo assinada.

\author{
Prof. Leonardo Lima Gomes \\ Orientador \\ Departamento de Administração - PUC-Rio
}

Pedro Americo Moretz-Shon David Co-Orientador Empresa de Pesquisa Energética - EPE

Prof. Luiz Eduardo Teixeira Brandão Departamento de Administração - PUC-Rio

Prof. Tara Keshar Nanda Baidya Departamento de Engenharia Industrial - PUC-Rio

Prof. André Luís Marques Marcato Universidade Federal de Juiz de Fora - UFJF

Luiz Guilherme Barbosa Marzano Centro de Pesquisas de Energia Elétrica - CEPEL

Prof. Nizar Messari Vice-Decano de Pós-Graduação do CCS

Rio de Janeiro, 03 de setembro de 2010. 
Todos os direitos reservados. É proibida a reprodução total ou parcial do trabalho sem autorização da universidade, do autor e do orientador.

\section{Marta Corrêa Dalbem}

Graduada em Engenharia Civil pela Universidade Federal do Rio de Janeiro (1983), Mestrado em Administração pela Coppead/UFRJ (1986). Experiência de 20 anos de trabalho em banco comercial multinacional; tem atuado em treinamento e consultoria a empresas, com foco em avaliação de projetos.

Ficha Catalográfica

Dalbem, Marta Corrêa

Análise de investimentos em energia eólica no Brasil / Marta Corrêa Dalbem ; orientador: Leonardo Lima Gomes ; co-orientador: Pedro Americo Moretz-Shon David. - 2010.

198 f. : il. (color.) ; $30 \mathrm{~cm}$

Tese (doutorado)-Pontifícia Universidade Católica do Rio de Janeiro, Departamento de Administração, 2010.

Inclui bibliografia

1. Administração - Teses. 2. Opções reais. 3. Jogos com opções reais. 4. Medida ômega. 5. Risco. 6. Energia eólica. 7. Leilões de energia. 8. Políticas de incentivo. I. Gomes, Leonardo Lima. II. David, Pedro Americo Moretz-Shon. III. Pontifícia Universidade Católica do Rio de Janeiro. Departamento de Administração. IV. Título. 
A Abol e Nyleide, que me deram raízes e asas.

A Adriano, Marina e Fernanda, luzes de minha vida. 


\section{Agradecimentos}

Ao meu orientador, professor Leonardo Lima Gomes, a quem serei para sempre grata pela generosidade com que me acolheu, pela orientação segura, pelo carinho e atenção com que sempre trata seus alunos e orientandos.

Ao meu co-orientador, professor Pedro Americo Moretz-Shon David, pela paciência e inestimável ajuda.

Aos professores André Luís Marques Marcato, Luiz Guilherme Barbosa Marzano, Tara Keshar Nanda Baidya, Luiz Eduardo Teixeira Brandão, por aceitarem participar de minha banca e pelas contribuições valiosas a este trabalho.

Aos professores da PUC-Rio, em especial a Diana Macedo-Soares, Jorge Ferreira, Kátia Rocha, Marcelo Klotzle, Patrícia Tomei, Paulo César Motta, pelo apoio nestes anos de Doutorado.

Ao professor Marco Antônio Dias da PUC-Rio e, novamente, ao professor Leonardo Lima Gomes, que tão generosamente dividiram comigo seus conhecimentos e se tornaram minhas referências do que deve ser um Professor.

Aos meus colegas de Doutorado Carlos de Lamare Bastian Pinto e Graziela Fortunato pela amizade e apoio em momentos difíceis, e aos meus também colegas de Doutorado Maria Paula Valente, Renée Spinelli, Maribel Suarez, Henrique Silva, que tornaram estes anos de estudo prazerosos.

À PUC-Rio, Capes e Faperj pelo apoio financeiro.

A Deus, pela oportunidade de conhecer tantas pessoas especiais e pela ajuda neste caminho, como em tantos outros. 


\section{Resumo}

Dalbem, Marta Corrêa; Gomes, Leonardo Lima. Análise de Investimentos em Energia Eólica no Brasil. Rio de Janeiro, Tese de Doutorado, p. 198 - Departamento de Administração de Empresas, Pontifícia Universidade Católica do Rio de Janeiro

Este trabalho foca a política adotada em 2009 no Brasil para a contratação de energia eólica via leilões e seus impactos na decisão de investimento. Dividida em três partes conectadas entre si, esta tese primeiro discute as políticas de incentivo à energia eólica já adotadas no Brasil e em outros países, os benefícios e dificuldades da inserção eólica, identificando os problemas da atual política e propondo alterações. Na segunda parte, analisa-se a decisão sobre que quantidade ofertar em leilões de energia eólica no Brasil, e a que preço, considerando-se o risco do vento e as regras específicas para o segmento eólico adotadas em 2009. Usa-se como critério de decisão a medida Ômega (KEATING \& SHADWICK, 2002), medida de risco ainda pouco disseminada no mundo corporativo e em especial no segmento eólico. Conclui-se que o setor tem incentivos para participar dos leilões ofertando um pouco mais que a geração esperada do projeto, haja vista que as regras são assimétricas para as situações em que o projeto está desempenhando abaixo ou acima do esperado. O resultado é útil para legisladores e para potenciais investidores em geração eólica no Brasil. Finalmente, considerando-se que alguns projetos de menor potencial eólico sobrepujaram outros de maior viabilidade econômica no leilão, a terceira parte deste trabalho procura identificar até que ponto o cenário incerto e as diferentes visões sobre o futuro podem ter favorecido este resultado. Supondo-se que ganhadores e perdedores do leilão apresentam assimetrias não só em termos do investimento necessário para entrar no mercado e no valor de seus projetos-padrão, mas também na forma como prevêem que o mercado para energia eólica evoluirá no Brasil, a tese analisa este problema à luz da teoria de Jogos com Opções Reais, avaliando a decisão de investir em projetos eólicos em ambiente de incerteza e sob os efeitos de competição. Mais especificamente, usa-se como referencial teórico os trabalhos de Huisman (2001) e Pawlina \& Kort (2006) para duopólios assimétricos, estendendo-os de modo a considerar três assimetrias: assimetria no 
investimento necessário para entrar no mercado, no fluxo de valor de cada jogador (representado pelo valor dos projetos-padrão que comporão o portfólio de cada jogador), assimetria no processo estocástico utilizado para caracterizar as perspectivas futuras para os projetos eólicos. Análises de sensibilidade do modelo aos parâmetros identificam as situações limite em que se passa a favorecer a entrada no mercado de projetos menos viáveis economicamente e, portanto, com menores chances de serem concretizados. Conclui-se que pequenas discrepâncias na visão de futuro podem ter deixado de fora do leilão empresas com maiores chances de materializarem seus projetos. $\mathrm{O}$ risco de preempção de empresas menos viáveis cai quando há menos informação sobre as crenças dos concorrentes, porém ao custo de preços de energia um pouco mais elevados para os consumidores. Observa-se que quando há baixa incerteza e similaridade de crenças, fruto de política governamental mais clara, favorece-se tanto uma tarifa baixa de energia quanto uma indústria eólica mais forte no Brasil.

\section{Palavras- Chave}

Opções Reais; Jogos com Opções Reais; Medida Ômega; Risco; Energia Eólica; Leilões de Energia; Políticas de Incentivo; Brasil. 


\section{Abstract}

Dalbem, Marta Corrêa; Gomes, Leonardo Lima. Analysis of Investments in Wind Power in Brazil. Rio de Janeiro, Doctoral Dissertation, 198 p.Departamento de Administração de Empresas, Pontifícia Universidade Católica do Rio de Janeiro

This dissertation focuses on the governmental policy adopted in 2009 to contract wind power energy in Brazil through auctions and its impacts on the decision to invest in the wind power industry, and is organized into three separate but interconnected studies. The first study discusses the incentive policies adopted in Brazil and worldwide and the benefits and difficulties of increasing the stake of wind power in the country, identifying potential problems in the current policy and proposing changes. In the second part, the decision on the optimal amount of power to offer at auctions - and at what price - is analyzed based on the wind risk at the project's site and on the specific rules for wind power in Brazil that were prevailing in 2009. For this purpose, the Omega measure (KEATING \& SHADWICK, 2002) was adopted as decision criterion. The use of the Omega measure is still new to the corporate world and has not yet been used in the wind industry. The main conclusion is that entrepreneurs have incentives to sell slightly more than the expected output of the wind farm, given that the purchasing rules are asymmetric for situations in which a farm is underperforming or is above expectations. This result is useful for legislators and potential investors in wind power generation in Brazil. Finally, considering that some projects with lower wind potential have won over others with higher potential, the third part of this work attempts to identify to what extent the uncertain scenario and investors' different outlooks of the market's potential may have influenced this result. Assuming that losers and winners of the auction had asymmetries not only in terms of the investments necessary to enter the market and build their portfolio of wind farms, but also in terms of their views on how the market for wind power will evolve in Brazil, this dissertation analyses this problem in light of the OptionGames Theory, deriving the decision to invest in wind projects under uncertainty and competition. The theoretical frameworks of this analysis are the works by Huisman (2001) and Pawlina \& Kort (2006) for asymmetric duopolies, extending 
their theory to consider three asymmetries: asymmetry in the investment to enter the market, in the value of each player's portfolio, and asymmetry in the stochastic process adopted by each player to describe the market prospects for wind farms. A sensitivity analysis to the parameters of the model identified the thresholds above which less viable firms preempt the market. The main conclusion is that minor asymmetries in the views about the future may have been sufficient to make more viable firms quit the auction earlier. The risk of less viable firms preempting the market drops when their opponent's beliefs are not known, but at the expense of higher energy prices for consumers. When uncertainty is low and beliefs are symmetric, a feasible scenario when the governmental policy for the sector is clearer, the result can be lower energy tariffs as well as a stronger wind industry in Brazil.

\section{Keywords}

Real Options; Option-Games; Omega Measure; Risk; Wind Energy; Energy Auctions; Support Schemes; Brazil. 


\section{Sumário}

1 Introdução 19

1.1. Organização da Tese 22

1.2. Revisão de Literatura 23

2 Análise das Políticas de Incentivo a Eólicas e das Novas Regras

Adotadas no Brasil em 2009, à luz da experiência em outros países 25

2.1. Introdução 25

2.2. Geração de Energia no Brasil - Breve Histórico e Contextualização 27

2.3. Riscos e Benefícios de Aumentar a Inserção da Geração Eólica 30

2.4. Histórico dos Incentivos a Eólicas, no Brasil 37

2.5. Novas Regras para a Geração Eólica - 2009

2.6. Comparação com as Políticas para Energia Eólica Adotadas em Outros Países 46

2.6.1. Incentivos à Implantação de Parques Eólicos na Europa 46

2.6.2. Alemanha 48

2.6.3. Reino Unido 48

2.6.4. Espanha $\quad 50$

2.6.5. Holanda $\quad 50$

2.6.6. França e Irlanda

2.6.7. China 51

2.6.8. Índia 53

2.6.9. EUA 53

2.7. Conclusões e Recomendações 54

2.8. Referências Bibliográficas 58

3 Decisão sobre como Ofertar Energia Eólica nos Leilões do Brasil, Considerando o Risco do Vento 63

3.1. Introdução 63

3.2. Revisão de Literatura 65 
3.2.1. Avaliação de Projetos

65

3.2.2. Medidas de Risco e Escolha de Carteiras

3.3. Leilões de Energia no Brasil e Regras de Contratação do Leilão 2009 para Eólicas

71

3.3.1. Histórico dos Leilões de Energia 71

3.3.2. Regras de Contratação do Leilão de Eólica 2009

3.4. Fluxo de Caixa: Premissas Adotadas 75

3.4.1. Investimento (Capex) 76

3.4.2. Quantidade Gerada de Energia e Receita de Vendas 77

3.4.3. Custos de Operação e Manutenção 77

3.4.4. Custos de Transmissão e Distribuição 78

3.4.5. Receitas de Créditos de Carbono 78

3.4.6. Despesas Financeiras 78

3.4.7. Tributação 78

3.4.8. Custo de Capital $\quad 79$

3.4.9. Resumo das Premissas Adotadas 80

3.5. Simulações dos Ventos e dos VPLs dos Parques Eólicos $\quad 81$

3.6. Decisão de Contratação com Base na Maximização da Medida Ômega 83

3.6.1. O Problema a Ser Resolvido 83

3.6.2. Exemplo Numérico 84

3.7. Conclusões 92

3.8. Referências Bibliográficas 94

4 Leilões de Energia Eólica no Brasil: Análise dos Resultados com Base na Teoria de Jogos com Opções Reais 105

4.1. Introdução 106

4.2. Revisão de Literatura 109

4.2.1. Opções Reais 109

4.2.2. Teoria dos Jogos 113

4.2.2.1. Caracterização do Jogo 114

4.2.2.2. Solução do Jogo 116

4.2.3. Jogos com Opções Reais (Option-Games) 118 
4.2.3.2. Solução de Jogos com Opções Reais Envolvendo Duopólios Assimétricos

4.3. Incertezas do Mercado de Energia Eólica no Brasil e Possíveis Condições de Assimetria entre os Concorrentes

4.4. O Problema, Modelado Como Um Duopólio Em Que Há Três Assimetrias

4.4.1. Premissas Básicas

129

4.4.2. Cenários/Modelos Considerados

135

4.4.3. Modelo 1 - Ambas Adivinham Corretamente

136

4.4.3.1. Empresa $i$ é o Líder, Empresa $j$ é o Seguidor 136

4.4.3.2. Empresa j é o Líder, Empresa $i$ é o Seguidor 143

4.4.4. Modelo 2 - Ambas Adivinham Erroneamente - o Impacto da Falta de Informação Sobre as Crenças do Concorrente

4.4.4.1. Empresa $i$ como Líder, Empresa j como Seguidor 145

4.4.4.2. Empresa $j$ como Líder, Empresa $i$ como Seguidor 146

4.5. Aplicação do Modelo e Discussão dos Resultados 146

4.5.1. Aplicação do Modelo 146

4.5.2. Discussão dos Resultados 150

$\begin{array}{ll}\text { 4.5.2.1. Cenário-Base } & 150\end{array}$

4.5.2.2. Modelo 1

$\begin{array}{lr}\text { 4.5.2.3. Modelo } 2 & 159\end{array}$

4.6. Conclusões e Recomendações 166

$\begin{array}{lr}\text { 4.7. Referências Bibliográficas } & 168\end{array}$

5 Conclusões e Recomendações para Futuras Pesquisas 180

6 Referências Bibliográficas Consolidadas 


\section{Lista de figuras}

Figura 2.1: Impacto de Eólicas e Outras Fontes Renováveis no Preço da Energia, Conforme o Nível dos Reservatórios das Hidrelétricas

Figura 2.2: Velocidade dos Ventos em Praia das Neves-ES, versus

Demanda Média Total por Energia Elétrica ao Longo do Dia, no Brasil

Figura 2.3: Geração Eólica Potencial na Região NE, versus a Vazão Natural do Rio São Francisco no Reservatório da Hidrelétrica de Sobradinho

Figura 2.4: Geração Eólica que Seria Obtida via Parque Eólico de 50 MW em Palmas, Comparado com a Geração Hidrelétrica na Região SE

Figura 2.5: Balanço Energético do Subsistema NE

Figura 2.6: Balanço Energético do Subsistema $S$

Figura 2.7: Balanço Energético do Subsistema SE-CO

Figura 2.8: Principais Geradoras de Energia Elétrica no Brasil e Parques

Eólicos PROINFA 1 (ago 2009)

Figura 2.9: Evolução da Política de Apoio a Fontes Renováveis na Europa, por Tipo de Incentivo, e Índice de Eficácia de Cada País para Eólicas On-shore 47 Figura 2.10: Remuneração da Energia Eólica na Europa, em Euros/ MWh, em 2006

Figura 3.1: Exemplo de Cálculo da Medida Ômega, Considerando-se que a Meta $L$ é Igual a 10.

Figura 3.2: Medida Ômega Como a Divisão de Dois Valores Esperados:

ganho esperado no trecho só de ganhos/perda esperada no trecho só de perdas $=\mathrm{EC}(\mathrm{L}) / \mathrm{ES}(\mathrm{L})$

Figura 3.3: Esquema de Comercialização dos Leilões de Energia de

Reserva (LER)

Figura 3.4: Exemplo de Parque Submetido às Regras do Leilão 2009.

Fonte: elaboração própria

Figura 3.5: Exemplo de Simulação da Série Sintética de Ventos e dos VPLs do Parque Eólico

Figura 3.6: VPL Médio Obtido nas 1000 Simulações, para Diferentes Níveis de Preço e Oferta. 
Figura 3.7: VPL Médio e VaR 95\%,99\% para o Caso EOL NE, ao Preço

R\$ 148/MWh.

Figura 3.8: Distribuição de VPLs no Caso EOL NE, Preço R\$ 148/MWh. 86

Figura 3.9: Decisão Ótima em Cada Critério; Caso EOL NE, Preço

R\$ 148/MWh.

Figura 3.10: EOL NE, Preço R \$ 148/MWh, Submetido a uma Série de

Ventos Fracos $\quad 88$

Figura 3.11: EOL NE, Preço R\$ 148/MWh, Submetido a uma Série de

Ventos Fortes

Figura 3.12: Medida Ômega, prob. VPL<0 e P90 para EOL NE, ao Preço de $\mathrm{R} \$ 148 / \mathrm{MWh}$

Figura 3.13: Curva dos Ômegas Ótimos para os Diversos Níveis de Preço

e os Respectivos Percentuais Ótimos.

Figura 4.1: Momento de Investir, Conforme Recomendado pelo Método do FCD e pela TOR

Figura 4.2: Valor de uma Empresa em Situação de Monopólio e já em

Situação de Duopólio, segundo o Método do Fluxo de Caixa Descontado.

121

Figura 4.3: Curva de Valor do Líder em um Duopólio.

Figura 4.4: Curvas de valor como Líder e como Seguidor de uma das

Empresas do Duopólio.

Figura 4.5: Entrada do Líder Quando não Há Ameaça de que o Oponente

Entrará Antes como Líder no Mercado

Figura 4.6: Curvas de Valor das Duas Empresas do Duopólio.

Figura 4.7: Distribuição de Frequências dos Ganhadores \& Perdedores do

Leilão 2009, por Garantia Física/Potência Instalada

Figura 4.8: Resultado das Projeções do FVE Esperado do Projeto-Padrão de

Cada Empresa, Quando Sujeitos à Variação no Preço Contratado da

Energia

Figura 4.9: Curvas de Valor no Cenário-Base, Horizonte de

Investimentos $=5$ anos

Figura 4.10: Curvas de Valor no Cenário-Base, Horizonte Infinito

de Investimentos

Figura 4.11: Curvas de Valor Quando o Cenário-Base é Alterado de 
Modo a Refletir Assimetria na Volatilidade dos FVEs, Horizonte=5

anos

Figura 4.12: Curvas de Valor Quando o Cenário-Base é Alterado de

Modo a Refletir Assimetria na Tendência dos FVEs.

Horizonte $=5$ anos

Figura 4.13: Curvas de Valor Quando o Cenário é Equivalente ao da

Figura 4.12, Porém Alterado de Modo a Refletir Maior Assimetria no Custo de Entrada no Mercado

Figura 4.14: Curvas de Valor Quando o Cenário é Equivalente ao Cenário-Base, Exceto no Que se Refere ao $\alpha j$, Agora Arbitrado em -5\%

Figura 4.15: Curvas de Valor Quando o Cenário é Equivalente ao Cenário-Base,

Exceto no que se Refere ao $\alpha i$, Agora Arbitrado em -29\%

Figura 4.16: Parâmetros Que Influenciam as Equações de Valor Como

Líder e Como Seguidor, Para Diferentes Valores de $\alpha$ e $\sigma$

Figura 4.17: Curvas de Valor Quando: (a) os Jogadores Conhecem a Visão

de Futuro do Competidor; (b) Cada Jogador Assume Que o Competidor

Tem a Mesma Visão de Futuro do Competidor; (b) Cada Jogador Assume

Que o Competidor Tem a Mesma Visão de Futuro que a Sua Própria

Figura 4.18: Gatilho para Investir, Como Monopolista ou Como Líder,

e Respectivos Valores em Cada Caso (Miopia de Leahy) 


\section{Lista de tabelas}

Tabela 2.1: Capacidade Instalada de Geração de Energia Elétrica no Brasil, Por Fonte 26

Tabela 3.1: Resumo das Premissas Adotadas $\quad 80$

Tabela 4.1: Resumo das Características de Cada Empresa do Duopólio: 133

Tabela 4 .2: Cenários a Serem Modelados 135

Tabela 4.3: Cenário-Base para Cada Empresa, no Qual não Há Assimetria Quanto

à Visão de Futuro 149

Tabela 4.4: Comparando o Modelo 1 e o Modelo 2, Para um Conjunto

Específico de Parâmetros 162

Tabela 4.5: Resultados Previstos pelos Modelos 1 e 2, em Diversos Cenários 164

Tabela 4.6: Resumo dos Resultados, Para Diferentes Visões de Futuro

dos Jogadores 165 


\section{Lista de Siglas e Abreviaturas}

ABEeólica - Associação Brasileira de Energia Eólica

$A C L$ - Ambiente de Contratação Livre

ACR - Ambiente de Contratação Regulada

AFRRM - Adicional de Frete para a Renovação da Marinha Mercante

AMC - Análise Multi-Critério

ANEEL - Agência Nacional de Energia Elétrica

ARIMA - Auto-Regressive Integrated Moving Average

ARMA - Auto-Regressive Moving Average

AWEA - American Wind Energy Association

BNB - Banco do Nordeste do Brasil

BNDES - Banco Nacional de Desenvolvimento Econômico e Social

CALL - Opção de Compra

CanWEA - Canadian Wind Energy Association

CAPEX - Capital Expenditures - Investimentos de Capital (Ativo Fixo \& outros)

CAPM - Capital Asset Princing Model

CCEE - Câmara de Comercialização de Energia Elétrica

CEPEL - Centro de Pesquisas de Energia Elétrica

CER - Contratos de Energia de Reserva

COFINS - Contribuição para o Financiamento da Seguridade Social

CONER - Conta de Energia de Reserva

CONUER - Contrato de Uso de Energia de Reserva

EER - Encargo de Energia de Reserva

EWEA - European Wind Energy Association

FCD - Fluxo de Caixa Descontado

FCLA - Fluxo de Caixa Livre para o Acionista

FCLP - Fluxo de Caixa Livre do Projeto

FVE - Fluxo de Valor da Empresa

GWEC - Global Wind Energy Council

ICSD - Índice de Cobertura do Serviço da Dívida

ICMS - Imposto sobre Circulação de Mercadorias e Prestação de 
Serviços

IGPM - Índice Geral de Preços do Mercado

IOF - Imposto sobre Operações Financeiras (de Crédito, Câmbio e

Seguros)

IPCA - Índice de Preços ao Consumidor Ampliado

IPI - Imposto sobre Produtos Industrializados

IR - Imposto de Renda

LAJIDA - Lucro Antes dos Juros, Imposto de Renda, Depreciação \& Amortização

LER - Leilão de Energia de Reserva

MAE - Mercado Atacadista de Energia

MGB - Movimento Geométrico Browniano

MME - Ministério de Minas e Energia

MRM - Movimento de Reversão à Média

PASEP - Programa de Formação do Patrimônio do Servidor

$\mathrm{PCH}$ - Pequena Central Hidrelétrica

PDEE - Plano Decenal de Expansão de Energia

PIB - Produto Interno Bruto

PIS - Programa de Integração Social

PROINFA- Programa de Incentivo a Fontes Alternativas

SEB - Setor Elétrico Brasileiro

SIN - Sistema Interligado Nacional

SMC - Simulação de Monte Carlo

S\&P - Standard \& Poor's

TJLP - Taxa de Juros de Longo Prazo

TOR - Teoria das Opções Reais

UNFCCC - United Nations Framework Convention on Climate Change,

Convenção Quadro das Nações Unidas sobre Mudança do Clima

VaR , V@R - Value At Risk

VETEF - Valor Econômico de Tecnologia Específica

VPL - Valor Presente Líquido

WWEA - World Wind Energy Association 